\title{
ÉTICA EM PESQUISA COM SERES HUMANOS NA WEB: O CASO DA PLATAFORMA BRASIL
}

\author{
ÉTICA EN LA INVESTIGACIÓN CON SERES HUMANOS \\ EN LA RED: EL CASO DE BRASIL PLATAFORMA
}

\author{
Nelma Camelo de Araujo * \\ Deise Juliana Francisco**
}

\begin{abstract}
RESUMO
Introdução: As Tecnologias da Informação e Comunicação possibilitam a aproximação entre diferentes pessoas, organizações e instituições de pesquisa. Nesse cenário e visando auxiliar o pesquisador das instituições de pesquisa no Brasil foi implantado o Sistema de Informação na web via Conselho Nacional de Saúde denominado Plataforma Brasil, que será discutido ao longo desse artigo.

Objetivo: Essa discussão visa despertar o pesquisador de qualquer área do conhecimento não apenas da área da saúde, da importância no uso da Plataforma quando da apresentação de seus projetos de pesquisa nos Comitês de Ética em Pesquisa de suas instituições de origem.

Metodologia: $\mathrm{O}$ artigo foi desenvolvido partindo da experiência das autoras enquanto representantes de Comitê de Ética em Pesquisa com Seres Humanos e suas percepções quanto as dificuldades encontradas por pesquisadores no uso da Plataforma Brasil.
\end{abstract}

Resultados: Possibilidade de uso da Plataforma Brasil como fonte de informação na busca por pesquisas nas diversas áreas do conhecimento no Brasil.

Conclusões: Apontam-se pontos positivos e negativos da informatização do sistema Comissão de Ética em Pesquisa e Conselho Nacional em Pesquisa - CEP/CONEP.

Palavras-chave: Ética em Pesquisa. Plataforma Brasil.

*Doutoranda no Programa de Pós-Graduação em Ciência da Informação da Universidade Federal de Santa Catarina. E-mail: nelmacamelo@gmail.com

** Professora Adjunto III no Centro de Educação da Universidade Federal de Alagoas. Coordenadora do Comitê de Ética em Pesquisa da UFAL desde 2011. E-mail: deisej@gmail.com 


\section{INTRODUÇÃO}

A tecnologia de Comunicação e Informação aproximou pessoas, instituições sociais, organizações públicas e privadas. Além de possibilitar uma interação com as diversas áreas de pesquisa no Brasil e no Mundo.

Os meios de socialização das informações ao redor do mundo podem ser percebidos, por meio das redes sociais, tais como: temos os repositórios institucionais e ferramentas que possibilitam Twitter, Facebook, Fucker, Youtube, Products e outros, na área das instituições de pesquisas o armazenamento de trabalhos produzidos na instituição que possibilitam realizar estatísticas e estudos sobre os indicadores de produtividade e de financiamento para futuros projetos de áreas especificas.

Assim o monitoramento de pesquisas acadêmicas ou demais instituições de pesquisa podem se dar por meio desse universo informacional, possibilitando uma melhor visibilidade da produção dos trabalhos produzidos em todas as áreas de conhecimento.

Este artigo objetiva refletir sobre a implantação da Plataforma Brasil e seus efeitos no processo de avaliação ética de protocolos de pesquisa brasileiros. Tal discussão é relevante no contexto de maior uso de tal dispositivo on line por pesquisadores e pelo contexto de discussão sobre o processo de análise ética de protocolos de pesquisa por parte do sistema das Comissões Ética de Pesquisa e o Conselho Nacional em Pesquisa CEP/CONEP. As indagações sobre o funcionamento desse sistema são muitas e geradas, em boa parte, pela perspectiva da pesquisa experimental que permeia os marcos legais sobre regulamentação ética em pesquisa em nosso país. Os pesquisadores de matriz qualitativas têm se manifestado sobre a inadequação das Resoluções e mesmo da Plataforma Brasil para análise e encaminhamento ético das pesquisas.

Assim, é este contexto que refletimos aqui, sobre o sistema informatizado de acompanhamento das pesquisas brasileiras. A implicação das autoras como membros de Comitê de Ética em Pesquisa - CEP, e também 
como pesquisadoras auxiliam a reflexão, na medida em que há contato diário com a Plataforma e com pesquisadores que a utilizam.

\section{2 ÉTICA EM PESQUISA COM SERES HUMANOS}

A ética em pesquisa com seres humanos tem por objetivo preservar aos participantes da pesquisa, sua integridade física, moral e social, pois se considerarmos que muitos trabalhos são realizados com comunidades menos favorecidas, como a população indígena, moradores de favelas, crianças em situação de risco, dentre outros, se não houvesse orientações éticas para se trabalhar com essas comunidades, estaríamos voltando no tempo, donde no período da Segunda Guerra Mundial eram realizadas atrocidades envolvendo seres humanos nas pesquisas sem critério ético.

De acordo com Bernardes (2010), as experiências antiéticas nesse período incluem vinte e três médicos que foram condenados por praticarem dentre outras atrocidades em nome da ciência as citadas pela a autora:

Colocar prisioneiros em câmaras de pressão, modificar a pressão atmosférica e observar suas mortes; estudar o impacto sobre o corpo humano da imersão em água gelada por períodos prolongados; infectá-los com tifo, malária e outras doenças para testar drogas e vacinas; esterilização e castração procurando métodos eficazes para esterilização em massa; administrar venenos para estudar seus efeitos letais; e estudos com gêmeos, infectando um, matando os dois e comparando os corpos em autópsia (BERNARDES, 2010, p.18).

No contexto da regulamentação sobre ética em pesquisa, a construção das orientações que permearam o tema está voltada para a área da saúde, pois a implicação de projetos com seres humanos a priori necessita de uma análise minuciosa sobre os participantes da pesquisa, possibilitando sua proteção e integridade, em experiências científicas - a qual deu origem ao Código de Nuremberg.

[...] elaborado após o julgamento dos criminosos nazistas, tornou-se o primeiro documento internacional relevante na regulamentação das pesquisas clínicas e influenciou as diretrizes que se seguiram. Suas referências foram as diretrizes 
alemãs anteriores à guerra que orientavam a ética médica e a experimentação científica (JACOMÉ, 2013, p.31).

Assim, após esse período de atrocidades várias orientações sobre os princípios éticos em pesquisas com seres humanos foram estabelecidos no mundo, cada país dispõe sobre uma legislação ou leis especificas sobre o assunto, permitindo um maior grau de confiabilidade das pesquisas, bem como os resultados éticos advindo de suas investigações, pois existe uma estrutura que avalia esses trabalhos antes de serem executados, mesmo que em alguns casos os próprios pesquisadores questionem os procedimentos exigidos, principalmente o Termo de Consentimento Livre de Esclarecimento - TCLE, quando uso de dados secundários.

Segundo a Resolução 466/2012 do Conselho Nacional de Saúde, o respeito devido à dignidade humana exige que toda pesquisa se processe após consentimento livre e esclarecido e, no caso de crianças e adolescentes ou legalmente incapaz também do assentimento dos sujeitos, indivíduos ou grupos que por si e/ou por seus representantes legais manifestem a sua anuência à participação na pesquisa.

As discussões acerca da legitimidade dos trabalhos acadêmicos, pesquisas em cursos de pós-graduação e outros, inclusive são questionados pela própria Coordenação de Aperfeiçoamento de Pessoal de Nível Superior Capes, apontando que inúmeros trabalhos são plágio ou auto plágio, dessa forma os CEPs tem responsabilidade em garantir que os trabalhos realizados com seres humanos, independente da área sigam os procedimentos éticos estabelecidos na legislação vigente, o problema é quando essa mesma legislação deixa lacunas sobre termos que são importantes para que se faça cumprir a aplicação ética nos trabalhos apresentados.

$O$ fato de que a legislação foi elaborada tendo como foco pesquisas voltadas para a área da saúde, que envolvam seres humanos na sua fragilidade, doenças, epidemias, fármacos dentre outros, as demais áreas como as Ciências Humanas e Sociais discutem o rigor da legislação no campo de atuação das pesquisas na área. 
A matriz de avaliação da ética em pesquisa com seres humanos no Brasil é predominantemente biomédica. Conceitos como riscos e benefícios, devolução dos resultados de pesquisa, benefícios compartilhados, termo de consentimento livre e esclarecido ou reparação por danos compõem o vocabulário compartilhado dos comitês de ética para avaliar projetos de pesquisa (DINIZ, 2008, p.421).

Os órgãos regulamentadores em pesquisa reconhecendo a necessidade de ouvir a área de Ciências Humanas e Sociais aprovou a Resolução CNS 510/16. Essa Resolução atende as demandas da área, permitindo análise diferenciada nos protocolos de pesquisas, principalmente no que tange aos riscos e a aplicação do Termo de Consentimento Livre Esclarecido.

Quando citamos a legislação que permeia o sistema CEP/CONEP, e as questões sobre Ética em Pesquisa com Seres Humanos, nos pautamos principalmente na Resolução do Conselho Nacional de Saúde a Resolução CNS 466/2012, que orienta quais são os procedimentos éticos a serem adotados em pesquisa com seres humanos.

Um dos princípios norteadores dessa Resolução é o Termo de Consentimento Livre Esclarecido, sendo assinado pelos sujeitos participantes da pesquisa. A área das Ciências Humanas e Sociais questionavam a forma de obter esse Consentimento, pois o formato que a Resolução 466/12 orienta que o Consentimento deve ser impresso em três vias, uma das cópias fica com o sujeito, uma com o pesquisador e uma na instituição responsável pela pesquisa. Com a Resolução da área das Ciências Humanas e Sociais, o TCLE poderá ser realizado de diversas formas, como? Por meio de vídeo, de gravação e outros, de acordo com os sujeitos participantes da pesquisa.

Diante do exposto, todas as áreas cientificam se valem da avaliação de seus pares sobre seus trabalhos de pesquisa, na construção dessas avaliações as instituições de pesquisas até a pouco tempo trabalhavam com protocolos (processos) de pesquisa físicos. Como isso o tempo de avaliação e o volume de documentos que eram analisados dificultava a agilidade em se dar respostas rápidas sobre a situação dessas pesquisas. 
Com a institucionalização da Plataforma Brasil, esses protocolos podem ser analisados pelos pesquisadores dos CEPs com mais rapidez, pois os pareceres são armazenados na própria plataforma.

\subsection{Sistema CEP/CONEP}

A relevância da formação de Comitês de Ética em Pesquisa (CEP) nas Instituições de Ensino Superior no Brasil é discutida principalmente por Maluf (2007), Diniz (2008), Tomanik (2008) e Jacomé (2013) como sendo uma Comissão importante, tanto pelo seu papel educativo, quanto de análise e acompanhamento dos protocolos de pesquisa. Ressalta-se que os CEP atuam na formação de pesquisadores para analisarem protocolos de pesquisas, bem como na capacitação de estudiosos de cursos de graduação e pós-graduação e a comunidade acadêmica no entendimento dos procedimentos éticos em pesquisa de acordo com a legislação vigente, qual seja, a Resolução do Conselho Nacional de Saúde n 466/2012.

O CEP é um órgão independente na sua atuação, tendo vinculação com o Conselho Nacional em Pesquisa - CONEP e com a instituição que o acolhe. Normalmente tais instituições são estabelecimentos de ensino, hospitais, ou seja, instituições que tratam de pesquisa em seu cotidiano. No Quadro 1, são expostas as atribuições de cada uma das instâncias (instituição que acolhe e Conep): 
Quadro 1 - Vínculos do Comitê de Ética em Pesquisa e responsabilidade de cada instância (BRASIL, 1996)

\begin{tabular}{|c|c|}
\hline INSTITUICío & CONEP \\
\hline $\begin{array}{l}\text { Organização e funcionamento do } \\
\text { CEP }\end{array}$ & $\begin{array}{l}\text { Credenciamento e suporte técnico } \\
\text { para o CEP }\end{array}$ \\
\hline 1. Fornecer infraestrutura fisica; & $\begin{array}{l}\text { 1. Receber a documentação } \\
\text { encaminhada pela instituiçăo e avaliar }\end{array}$ \\
\hline $\begin{array}{l}\text { 2. Prover recursos humanos e } \\
\text { materiais; }\end{array}$ & a solicitaçăo feita; \\
\hline $\begin{array}{l}\text { 3. Definir critérios para tempo de } \\
\text { mandato e escolha dos membros; }\end{array}$ & $\begin{array}{l}\text { 2. Proceder ao credenciamento do } \\
\text { CEP: }\end{array}$ \\
\hline 4. Indicar os membros; & $\begin{array}{l}\text { 3. Comunicar a decisăo à direçăo da } \\
\text { instituiçăo; }\end{array}$ \\
\hline $\begin{array}{l}\text { 5. Encaminhar documentaçăo para } \\
\text { a Conep: criação do CEP, renovação } \\
\text { do credenciamento e substituição de } \\
\text { membros; }\end{array}$ & $\begin{array}{l}\text { 4. Fornecer material instrucional } \\
\text { (resoluçōes, manuais, publicaçōes, } \\
\text { material educativo) para promover } \\
\text { o funcionamento do CEP e o } \\
\text { treinamento de seus membros; }\end{array}$ \\
\hline $\begin{array}{l}\text { 6. Propiciar mecanismos que garantam } \\
\text { liberdade e independência para o } \\
\text { funcionamento do CEP; }\end{array}$ & $\begin{array}{l}\text { 5. Funcionar como instância recursal } \\
\text { do CEP em caso de litigio; }\end{array}$ \\
\hline $\begin{array}{l}\text { 7. Elaborar politicas institucionais } \\
\text { para: fomento à pesquisa, proteçăo } \\
\text { aos participantes e capacitaçăo e } \\
\text { treinamento de membros do CEP bem } \\
\text { como de pesquisadores da instituiçăo. }\end{array}$ & $\begin{array}{l}\text { 6. Avaliar o desempenho do CEP } \\
\text { por meio dos relatórios trimestrais } \\
\text { recebidos; } \\
\text { 7. Revisar os protocolos de pesquisa } \\
\text { de áreas temáticas especiais. }\end{array}$ \\
\hline
\end{tabular}

Fonte: GUILHEM e GRECO (2008, p. 101)

Para agilizar essas avaliações e principalmente para atender a demanda dos pesquisadores o Conselho Nacional de Saúde, criou em por meio da resolução 196/1996 a Comissão Nacional de Ética em Pesquisa - CONEP, que tem como finalidade:

A CONEP tem como principal atribuição o exame dos aspectos éticos das pesquisas que envolvem seres humanos. Como missão, elabora e atualiza as diretrizes e normas para a proteção dos sujeitos de pesquisa e coordena a rede de Comitês de Ética em Pesquisa das instituições.

Cabe a CONEP avaliar e acompanhar os protocolos de pesquisa em áreas temáticas especiais com o: genética e reprodução humana; novos equipamentos; dispositivos para a saúde; novos procedimentos; população indígena; projetos ligados à biossegurança e como participação estrangeira. A CONEP também se constitui em instância de recursos para qualquer das áreas envolvidas ${ }^{1}$.

\footnotetext{
${ }^{1}$ http://www.conselho.saude.gov.br/Web_comissoes/conep/index.html
} 
Desde 2001 por meio do Sistema Nacional de Informação sobre Ética em Pesquisa - Sisnep, em parceria com alguns CEPs e a CONEP foi construído um banco de dados dos protocolos (processos) de pesquisas interligados, na perspectiva da avaliação desses protocolos. As finalidades do processo de informatização desses protocolos foram:

- Facilitar o registro das pesquisas envolvendo seres humanos e orientar a tramitação de cada projeto para que todos sejam submetidos à apreciação ética antes de seu início; - Integrar o sistema de avaliação ética das pesquisas no Brasil (CEPs e CONEP) e propiciar a formação de um banco de dados nacional;

- Agilizar a tramitação e facilitar aos pesquisadores o acompanhamento da situação de seus projetos; - Oferecer dados para a melhoria do sistema de apreciação ética das pesquisas e para o desenvolvimento de políticas públicas na área;

- Permitir o acompanhamento dos projetos já aprovados (em condições de serem iniciados) pela população em geral e, especialmente, pelos participantes nas pesquisas. (http://portal2.saude.gov.br/sisnep/).

Barbosa, Corrales e Silbermann (2014) afirmam que o sistema era limitado, na medida em que contemplava apenas as folhas de rosto dos protocolos e o resultado a revisão ética dos projetos, sem chegar a contemplar a totalidade dos CEP.

Com isso o Conselho Nacional de Saúde - CNS, percebendo as dificuldades encontradas pela CONEP e consequentemente os CEPs em responder os pesquisadores nas avaliações de seus protocolos de pesquisa, lançou em 2012 a Plataforma Brasil, um sistema online que acompanha todo os tramites dos protocolos de pesquisa apresentados por todas as áreas do conhecimento nas instituições de pesquisa no Brasil.

\section{PLATAFORMA BRASIL}

Participando da evolução das novas tecnologias nas instituições de pesquisa, a ferramenta apresentada pelo CNS, veio contribuir de forma efetiva no controle de informações sobre a produção da pesquisa em nível nacional, pois o CNS, CONEP e CEP conseguem obter informações sobre a produção 
das pesquisas realizadas por essas instituições que são apresentadas no Sistema.

Esta proposta segue iniciativas nacionais de implantação de plataformas on line para fomentar e viabilizar a comunicação e divulgação entre os órgãos públicos e os cidadãos. Neste sentido, Cavalcante (2014) contextualiza que:

A implantação dos sistemas virtuais se apresenta como um meio para que a Administração Pública consiga cumprir os preceitos encartados na Carta Magna, em especial o princípio da eficiência administrativa, a celeridade processual, o acesso aos órgãos públicos, publicidade dos atos administrativos e fiscalização dos mesmos pela sociedade, todos eles como corolário do exercício da cidadania (CAVALCANTE, 2014, p. 20)

A Plataforma Brasil tem como princípio permitir o acesso ao número de pesquisas aprovadas ou em tramitação, divididos por região/estados e por área. Além disso, contará também com um campo dedicado ao cadastro de pesquisadores e um espaço para os Comitês de Ética em Pesquisa (CEP) fazerem o acompanhamento das pesquisas tanto em âmbito local como aquelas em tramitação na CONEP. A sociedade poderá ter acesso ainda aos dados de todas as pesquisas aprovada, conforme informação no próprio sitio do CNS.

Figura 1 - Plataforma Brasil

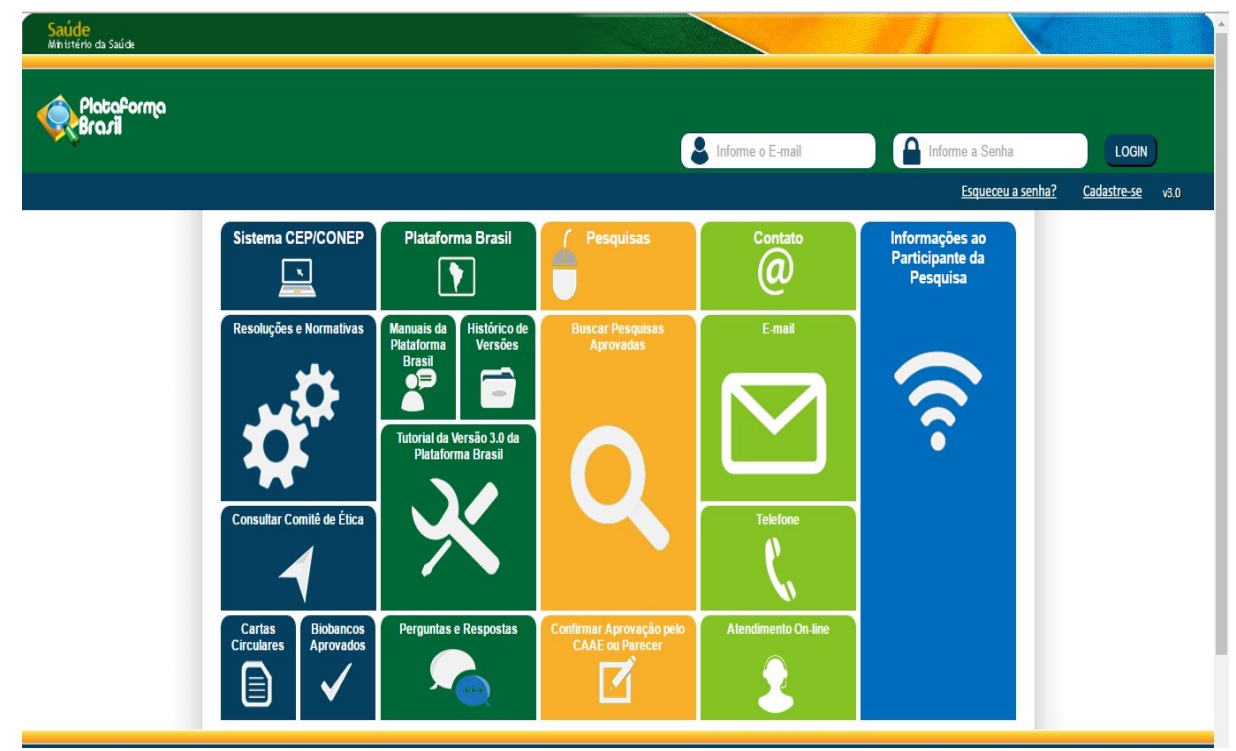

Fonte: http://aplicacao.saude.gov.br/plataformabrasil//ogin.jsf

Inf. Inf., Londrina, v. 21, n. 3, p. 361 - 375, set./dez., 2016. 
Quando o pesquisador se cadastra na Plataforma para inserir seu protocolo de pesquisa, a tela muda, assim, existe várias opções que dão acesso ao próprio pesquisador sobre o andamento de sua pesquisa. Também os CEPs e a CONEP, dispõe de acessos específicos, sendo monitorado o andamento dos protocolos. No caso do pesquisador que necessite visualizar se existe cadastro de pesquisas que lhe interessam, ou semelhantes a que ele está realizando seu trabalho, é possível visualiza-las se já foram disponibilizadas pelos CEPs e CONEP no sistema. Por meio da plataforma são gerados os indicadores de trabalhos por área de conhecimento e o andamento dos protocolos de pesquisas em cada CEPs espalhados nas instituições de pesquisa no Brasil. No quadro 2 é possível identificar a mudança de ambiente quando do cadastro do pesquisador e também perceber o acesso que a plataforma dispõe em diversas momentos.

Quadro 2 - Plataforma Brasil/ Acesso do Pesquisador

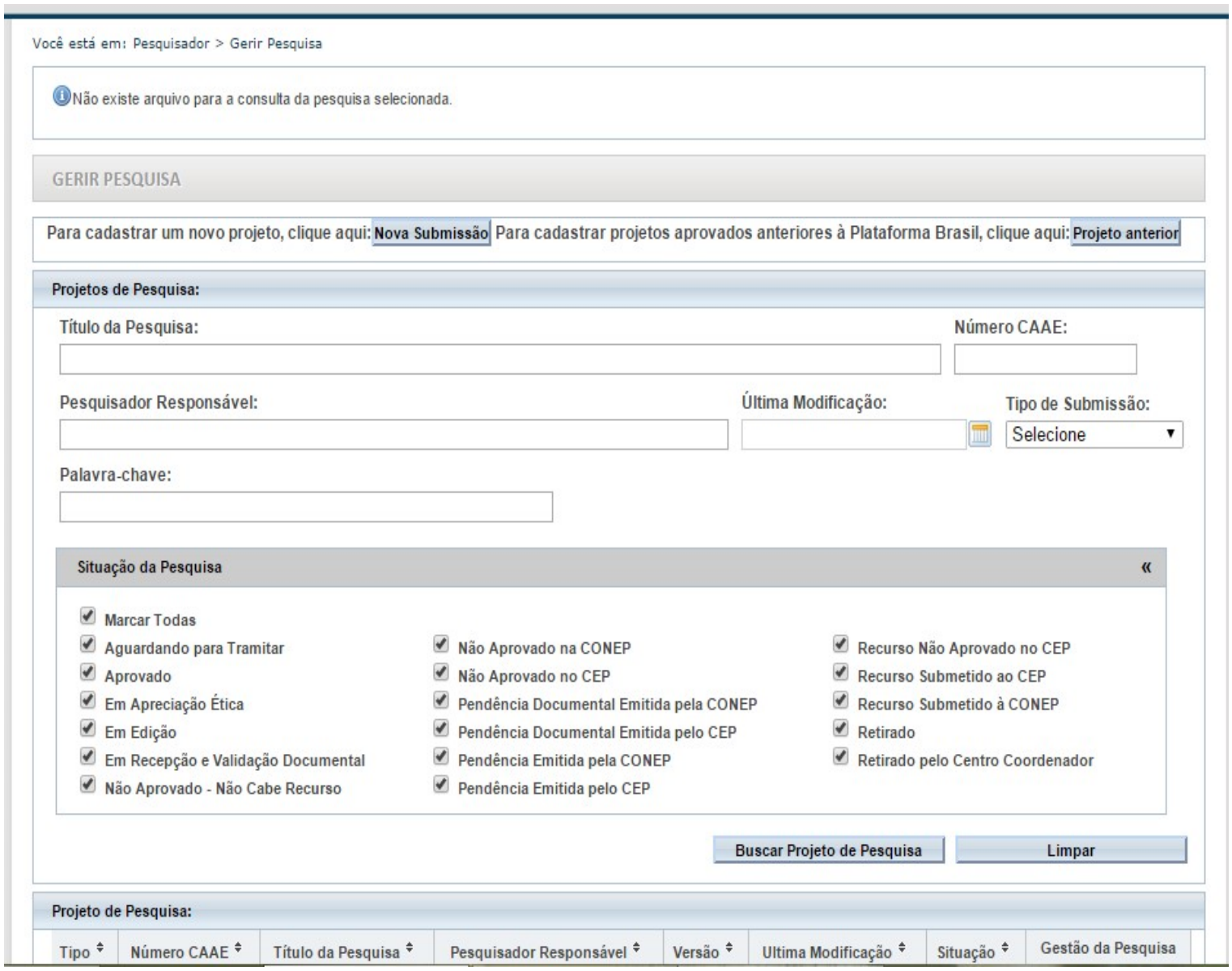

Fonte: http://aplicacao.saude.gov.br/plataformabrasil/visao/pesquisador/gerirPesquisa/gerirPesquisa.jsf 
Enfim, a plataforma é um meio de comunicação não apenas entre pesquisador e CEPs, mas também um veículo de disseminação de informações sobre as pesquisas realizadas nas instituições de pesquisa em nível nacional.

A Plataforma Brasil foi implantada quando ainda não estava totalmente preparada para uso nacional. Sendo assim, o processo de adaptação tem sido realizado no mesmo momento de suas atualizações. Neste sentido, a utilização pelos CEP e pesquisadores, no início, foi difícil e gradual.

A pergunta gerada por pesquisadores é sobre o próprio processo de análise ética sustentada pela Resolução 466/2012, tendo em vista que a plataforma é uma operacionalização do proposto na Resolução. As críticas ao modelo de base da análise ética ser baseada no modelo biomédico (pois a resolução ainda está pautada na área da saúde) mantêm-se.

A capacitação para uso da Plataforma é uma atividade educativa constante das atribuições dos CEPs e da CONEP, porém o que se percebe é que uma parcela significativa de pesquisadores da Instituições de Pesquisa no Brasil não apoia o sistema implantado, qual seja, a PLATAFORMA BRASIL, sendo difícil convencer esses pesquisadores da importância de seus protocolos de pesquisas na plataforma.

\subsection{Plataforma Brasil: fonte de informação}

Unindo o conceito da área da Ciência da Informação sobre Ética da Informação, termo cunhado por Capurro (1988), vislumbrando as questões relativas a ética na biblioteconomia, e fonte de informação, o modelo apresentado pelo sistema CEP/CONEP, agrega a atuação dos profissionais bibliotecários e os pesquisadores da área da Ciência da Informação.

Os profissionais bibliotecários no que tange a orientação junto aos CEPs na disseminação do uso da Plataforma por seus usuários e os pesquisadores na construção de projetos que venham a contribuir nas discussões sobre qual o papel da área relativa a Ética da Informação e seus desdobramentos interdisciplinares com as demais áreas que utilizam a Resolução 466/12 e também a Resolução 510/16. 
A discussão na área é insipiente, mas a possibilidade de projetos e atuação por parte dos profissionais da área da Ciência da Informação, como os bibliotecários e arquivistas se faz necessária.

Em uma breve consulta no site da BRACPI (2015), percebe-se que poucos periódicos solicitam que as pesquisas realizadas com seres humanos na área da Ciência da Informação, tenham a aprovação do Comitê de Ética em Pesquisa. Segue o resultado dos periódicos que informam a necessidade de se ter a aprovação do CEP: 1 ÁGORA: Revista do Arquivo Público do Estado de Santa Catarina. \& Curso de Arquivologia da Universidade Federal de Santa Catarina; 2 Revista Brasileira de Educação em Ciência da Informação RBECIN - periódico da Associação Brasileira de Ensino em Ciência da Informação - ABECIN; 3 RECIIS - Revista Eletrônica de Comunicação, Informação \& Inovação em Saúde; 4 LIINC em revista; 5 Perspectivas em Gestão \& Conhecimento da Universidade Federal da Paraíba; 6 Transinformação.

\section{REFLEXÃo}

Assim, a proposta do Sistema CEP/CONEP, tendo em vista agilizar a análise dos processos de pesquisas sem a consulta prévia aos pesquisadores, faz com que em várias situações os pesquisadores deixam de inserir documentos pertinente aos seus projetos, atrasando na mesma proporção a aprovação do mesmo, voltando a realidade dos processos físicos.

$\mathrm{Na}$ Plataforma existe a orientação de como o pesquisador deve proceder para inserir sua pesquisa no sistema e quais são as etapas a serem cumpridas, porém por falta de esclarecimentos in loco esses pesquisadores sem experiência no sistema duplicam informações, e por isso seus protocolos são devolvidos.

Os CEPs têm a responsabilidade de orientar os pesquisadores de suas respectivas instituições da importância de cadastrar seus projetos na Plataforma Brasil, quais são os procedimentos para tal e como se dá a análise de cada protocolo de pesquisa no sistema. 
Portando o Sistema CEP/CONEP, mais que normatizar os procedimentos éticos de pesquisa no Brasil, também tem a responsabilidade de conscientizar os pesquisadores da importância de manterem seus projetos na Plataforma Brasil, que possibilitará em curto prazo de tempo, uma fonte de informação poderosa na consulta sobre o andamento de pesquisas no Brasil, nas diversas áreas do conhecimento, possibilitando a atuação efetiva da área da Ciência da Informação no desenvolvimento de pesquisas que amplie a abordagem sobre Ética da Informação.

\section{REFERÊNCIAS}

BARBOSA, A.; CORRALES, C.; SILBERMANN, M. Controvérsias sobre a revisão ética de pesquisas em ciências humanas e sociais pelo Sistema CEP/CONEP. Rev. Bioét. (Impr). v.22, n.3, p.482-92.

BRAPCI. Base de Dados Referencial de Artigos de Periódicos em Ciência da Informação. Publicações. Curitiba: UFPR, 2015. Disponível em: < http://www.brapci.ufpr.br/ic.php?dd99=journals>. Acesso em: 13 jun. 2015.

BRASIL. MINISTÉRIO DA SAÚDE. Plataforma Brasil. Disponível em:< http://aplicacao.saude.gov.br/plataformabrasil/login.jsf > Acesso em: 20 mar. 2015.

. Conselho Nacional de Saúde. Disponível em:

< http://www.conselho.saude.gov.br/Web_comissoes/conep/index.html>. Acesso em: 20 mar 2015.

Sistema Nacional de Informações sobre Ética em Pesquisa envolvendo Seres Humanos. Disponível em:

<http://portal2.saude.gov.br/sisnep/>. Acesso em: 20 mar. 2015

CAPURRO, RI. Informationsethos und Informationsethik. Gedanken zumVerantwortungsvollen Handeln im Bereich der Fachinformation.

Nachrichten für Dokumentation, v. 39, p.1-4, 1988.

CONSELHO NACIONAL DE SAÚDE. Comissão Nacional de Ética em Pesquisa. Resolução 510 de abril de 2016. Disponível em: < http://conselho.saude.gov.br/resolucoes/2016/Reso510GM.pdf>. Acesso em 20 de abril de 2016. 
CAVANCANTE, F. PLATAFORMA BRASIL - uma visão crítica sob a ótica jurídica. In: FRANCISCO, D.J.; SANTANA, L (ORG). Problematizações éticas em pesquisa. Maceió: Edufal, 2014.

DINIZ, D. Ética na pesquisa em ciências humanas - novos desafios. Ciência \& Saúde Coletiva, Rio de Janeiro, v.13, n.2, p.417-426, 2008.

GUILHEM, D.; GRECO, D. A Resolução CNS 196/1996 e o Sistema CEP/Conep, In: DINIZ, D. et al. (Org.). Ética em pesquisa: temas globais. Brasília: Letras Livres, Ed. UNB, 2008

JACOMÉ, M. Q. D. Análise dos comitês de ética em pesquisa no Brasil: percepção de seus coordenadores e membros. 215f. 2013. Tese (Doutorado em Bioética). Programa de Pós Graduação em Bioética da Universidade de Brasília. Brasília, 2013.

MALUF, F. Compromisso dos pesquisadores com Comitês de Ética em Pesquisa nas dissertações e teses envolvendo seres humanos do Programa de Pós-Graduação em Ciências da Saúde da Universidade de Brasília no período de agosto 1996 - dezembro 2006. 86f. 2007.

Dissertação (Mestrado em Ciência da Saúde). Programa de Pós Graduação em Ciência da Saúde da Faculdade da Ciência da Saúde da Universidade de Brasília. Brasília. 2007.

TOMANIK, E.A. A Ética e os Comitês de Ética em Pesquisa com Seres Humanos. Psicologia em Estudo, Maringá, v. 13, n. 2, p. 395-404, abr./jun. 2008.

Title

Ethics in research with human beings on the web: the case of Brazil Platform

\begin{abstract}
Introduction: The Information and Communication Technologies - ICTs, enable rapprochement between different people, organizations and research institutions. In this scenario and in order to help researchers of research institutions in Brazil was implemented the Information System on the web through the National Health Council called Platform Brazil, which will be discussed throughout this article.

Objective: This discussion aims to awaken the researcher in any field of knowledge not only of health, the importance in using the platform when presenting their research projects in Ethics Committees in Research of their home institutions.

Methodology: The article was developed starting from the experience of the authors as representatives of the Research Ethics Committee and their perceptions about the difficulties encountered by researchers in the use of the Platform Brazil.

Results: Use of Possibility Platform Brazil as a source of information in the search for research in various fields of knowledge in Brazil.
\end{abstract}


Conclusions: They point to positives and negatives of the computerization of the Ethics Committee System Research and the National Research Council - CEP / CONEP.

Keywords: Research Ethics. Platform Brazil.

\section{Titulo}

Ética en la investigación con seres humanos en la red: el caso de Brasil Plataforma

\section{Resumen}

Introducción: Las Tecnologías de Información y Comunicación - TIC, permiten el acercamiento entre diferentes personas, organizaciones e instituciones de investigación. En este escenario y con el fin de ayudar a los investigadores de las instituciones de investigación en Brasil se implementó el Sistema de Información en la web a través del Consejo Nacional de Salud denominado Plataforma Brasil, que será discutido en este artículo.

Objetivo: Esta discusión tiene como objetivo despertar el investigador en cualquier campo del conocimiento no sólo de la salud, la importancia en el uso de la plataforma en la presentación de sus proyectos de investigación en los Comités de Ética en Investigación de sus instituciones de origen.

Metodología: El artículo fue desarrollado a partir de la experiencia de los autores como los representantes del Comité de Ética de Investigación y sus percepciones acerca de las dificultades encontradas por los investigadores en el uso de la Plataforma de Brasil.

Resultados: El uso de la plataforma Posibilidad Brasil como una fuente de información en la búsqueda de la investigación en diversos campos del conocimiento en Brasil.

Conclusiones: Señalan positivos y negativos de la informatización del Sistema de Investigación Comité de Ética y el Consejo Nacional de Investigación - CEP / CONEP.

Palabras clave: Ética de la investigación. Plataforma Brasil.

Recebido em: 10.04.2016

Aceito em: 18.11.2016 